\title{
INFLUÊNCIA DO ARMAZENAMENTO DE FOLHAS SECAS NO ÓLEO ESSENCIAL DE PATCHOULI (Pogostemon cablin BENTH.)
}

Trícia Cavalcanti Pergentino de Sant'ana, Arie Fitzgerald Blank*, Sylvia Dantas Vieira e Maria de Fátima Arrigoni-Blank Departamento de Engenharia Agronômica, Universidade Federal de Sergipe, Av. Mal. Rondon, s/n, 49100-000-São Cristóvão - SE, Brasil Hugo César R. de Jesus e Péricles Barreto Alves

Departamento de Química, Universidade Federal de Sergipe, Av. Mal. Rondon, s/n, 49100-000 São Cristóvão - SE, Brasil

Recebido em 30/7/09; aceito em 18/2/10; publicado na web em 10/6/10

\begin{abstract}
STORAGE INFLUENCE OF DRIED LEAVES ON PATCHOULY (Pogostemon cablin Benth.) ESSENTIAL OIL. The aim of this work was to evaluate the influence of five storage times of dry leaves of two patchouli genotypes on its essential oil content and chemical composition. Harvest was realized four months after planting. Storage influenced essential oil content of genotype POG-002. Patchoulol was the majority compound. Storage of dry leaves increased significatively the content of the compounds $\alpha$-bulnesene and germacrene A of genotype POG-021 and longicanfenilone, pogostol and patchoulol of POG-002. However, storage reduced significatively the content of the compounds cicloseichelene, $\beta$-cariofilene, $\alpha$-guaiene, acifilene and $\alpha$-bulnesene of the essential oil of genotype POG-002.
\end{abstract}

Keywords: Pogostemon cablin; Lamiaceae; patchouli genotypes.

\section{INTRODUÇÃO}

O patchouli, Pogostemon cablin Benth. (Lamiaceae), produz um óleo essencial extraído por destilação a vapor de suas folhas secas, ${ }^{1}$ que possui diversas propriedades, dentre elas aromaterápicas, ${ }^{2}$ atividade antibacteriana contra Escherichia coli, Staphylococcus aureus, Streptococus pyogenes, Bacterium coli, B. typhosum e Mycobacterium tuberculosis,${ }^{3}$ antioxidante,${ }^{4}$ inseticida, ${ }^{5}$ e repelente contra insetos. ${ }^{2,6}$

O óleo essencial de patchouli é largamente empregado em perfumaria e cosmética, como base e característica duradoura às fragrâncias, e como matéria-prima muito importante na fabricação de sabonetes, cosméticos, incensos, produtos de higiene oral e pós-barba. ${ }^{1,7,8}$ O óleo essencial de patchouli está entre os 18 óleos essenciais com maior importância comercial no mundo, e no Brasil a indústria Raros produz, beneficia e exporta 12 t/ano deste óleo essencial. ${ }^{9}$

A composição química, qualitativa e quantitativa, do óleo de patchouli varia bastante, sendo encontrada em diversos estudos uma grande variedade de compostos, com registros de 65 mono- e sesquiterpenos, sendo os sesquiterpenos a classe predominante na espécie. ${ }^{1,4,6,10-13}$ Nestes trabalhos os compostos com maior frequência de ocorrência foram os seguintes: $\beta$-elemeno, variando de $0,24-1,3 \%$, $\beta$-patchouleno $(0,94$ $12,12 \%), \beta$-cariofileno $(1,88-5,14 \%), \alpha$-patchouleno $(2,3-20,9 \%)$, $\alpha$-guaieno (3,17-22,20\%), seicheleno (4,73-8,94\%), $\alpha$-bulneseno $(9,86-20,30 \%)$ e patchoulol $(17,50-54,31 \%)$.

O principal composto do óleo de patchouli é o patchoulol, que é importante para a duração do seu odor, embora no mais alto estado de pureza química seja praticamente inodoro. ${ }^{11}$

O patchoulol e $\alpha$-patchouleno são os constituintes do óleo essencial de patchouli que regulam seu aroma. ${ }^{1} \mathrm{O}$ óleo essencial possui um forte odor balsâmico amadeirado, com nuances herbáceas e floral.

$\mathrm{Na}$ Indonésia um óleo essencial de patchouli com 35\% de patchoulol é frequentemente misturado com um óleo mais barato, com menor teor de patchoulol, importado da China, para reduzir o preço. ${ }^{8}$

Vários fatores podem causar variações na composição de óleos essenciais, tais como, efeitos ambientais (umidade, temperatura e luminosidade), cultivar ou origem do material, práticas agrícolas, maturidade e tratamentos pós-colheita. ${ }^{14}$ Para o patchouli vários fatores podem

*e-mail: afblank@ufs.br influenciar a composição do seu óleo essencial, tais como, a qualidade do material foliar, o método de destilação, o envelhecimento das folhas secas e do óleo essencial, ${ }^{7}$ a idade das plantas e práticas de cultivo. ${ }^{13}$ Foi constatado que vários constituintes do óleo essencial de patchouli são perdidos ou enriquecidos durante o processo de destilação comercial. ${ }^{15}$

Variações químicas também ocorrem entre materiais de patchouli provenientes de diferentes regiões, ${ }^{12}$ com os óleos de patchouli da China e da Indonésia diferindo entre si quanto ao teor de patchoulol. ${ }^{8}$ Já para horário de colheita, não foi observada influência na composição química do óleo essencial de patchouli cultivado em Botucatu-SP. ${ }^{16}$

Além disso, o teor e o rendimento do óleo essencial de patchouli também podem ser influenciados por fatores como adubação, irrigação, espaçamento, cultivar e cultivo em consórcio., ${ }^{1,17,18}$

A existência de quimiotipos, comum em espécies da família Lamiaceae ${ }^{14}$ também foi observada em plantas de $P$. cablin, com identificação de dois quimiotipos do óleo essencial de patchouli, o tipo pogostone e o tipo patchoulol. ${ }^{12,19}$ Desta forma, os componentes patchoulol e pogostone podem auxiliar no controle de qualidade deste óleo, ajudando a distinguir substitutos ou adulterantes. ${ }^{12}$ Além disso, também foi identificado um tipo intermediário entre estes dois quimiotipos. ${ }^{12}$

Devido à grande demanda e ao valor comercial do óleo essencial de patchouli, a existência de vários fatores que podem influenciar o teor, rendimento e a composição química do óleo essencial, este trabalho teve como objetivo avaliar a influência do tempo de armazenamento das folhas secas no teor, rendimento e composição química do óleo essencial de dois genótipos de patchouli (Pogostemon cablin Benth.) do Banco de Germoplasma da Universidade Federal de Sergipe.

\section{PARTE EXPERIMENTAL}

\section{Material vegetal}

O ensaio foi implantado na Fazenda Experimental "Campus Rural da UFS", localizada no município de São Cristóvão-SE, latitude $11^{\circ} 00^{\prime} \mathrm{S}$ e longitude $37^{\circ} 12^{\prime} \mathrm{W}$. Foram cultivados dois genótipos de Pogostemon cablin (POG-002 e POG-021) obtidos do Banco Ativo de Germoplasma (BAG) da Universidade Federal de Sergipe (UFS), e testaram-se 5 tempos de armazenamento das folhas secas $(0,3,7$, 14 e 28 dias) após secagem. O genótipo POG-002 (Voucher n⿳ํㅡㄹ 13170 
no Herbário da UFS) é material genético adquirido em uma feira de São Paulo-SP e o genótipo POG-021 (Voucher nº 13177 no Herbário da UFS) foi adquirido da empresa indiana Growmore Biotech Ltd.

O delineamento experimental foi em blocos ao acaso, em esquema fatorial $2 \times 5$, com 3 repetições e cada repetição foi composta por 6 plantas. O espaçamento usado foi de $0,50 \mathrm{~m}$ entre plantas e $0,50 \mathrm{~m}$ entre linhas, e a adubação aplicada foi de $6.000 \mathrm{~kg} \mathrm{ha}^{-1}$ de esterco bovino e $1.000 \mathrm{~kg} \mathrm{ha}^{-1}$ de NPK (6-24-12+micronutrientes). O experimento foi implantado em 27/5/2008 e colhido após 4 meses, com corte realizado a $25 \mathrm{~cm}$ do solo. $\mathrm{O} \mathrm{pH}$ do solo foi corrigido para saturação de bases de $80 \%$. Todas as folhas foram colhidas em 12/02/2009 e secas a $40 \pm 1$ ${ }^{\circ} \mathrm{C}$ por 4 dias em estufa de secagem com fluxo de ar forçado. As folhas foram armazenadas em sacos de polietileno preto de $100 \mathrm{~L}$, retirandose o ar com auxílio de um aspirador elétrico, dentro do Laboratório de Fitotecnia do Depto. de Engenharia Agronômica da UFS. A temperatura média dentro do laboratório foi de $32^{\circ} \mathrm{C}$.

\section{Extração e análise do óleo essencial}

O óleo essencial foi extraído pelo método de hidrodestilação em aparelho do tipo Clevenger, utilizando-se $50 \mathrm{~g}$ de folhas secas, expressandose os resultados em \% (baseada em $\mathrm{mL} 100 \mathrm{~g}^{-1}$ de peso da matéria seca).

A análise qualitativa da composição química do óleo essencial foi realizada por cromatógrafo a gás acoplado a espectrômetro de massas CG-EM (Shimadzu, modelo QP 5050A), equipado com um autoinjetor AOC-20i (Shimadzu) e coluna capilar de sílica fundida J\&W Scientific DB-5MS de $30 \mathrm{~m}$ x 0,25 mm d.i., 0,25 $\mu \mathrm{m}$ de filme, usando He como gás de arraste com fluxo de $1,2 \mathrm{~mL} \mathrm{~min}^{-1}$, a temperatura foi programada mantendo $50^{\circ} \mathrm{C}$ por 2 min, seguido de um aumento de $4{ }^{\circ} \mathrm{C} \min ^{-1}$ até atingir $200{ }^{\circ} \mathrm{C}$, depois a $15^{\circ} \mathrm{C}$ até atingir $300^{\circ} \mathrm{C}$ mantendo constante esta temperatura por $15 \mathrm{~min}$; temperatura do injetor de $250{ }^{\circ} \mathrm{C}$ e temperatura do detector de $280{ }^{\circ} \mathrm{C} ; 40 \mathrm{mg}$ do óleo foi diluído em 1,5 mL de acetato de etila e injetado um volume de $0,5 \mu \mathrm{L}$ da solução; taxa de partição do volume injetado de 1:100 e pressão na coluna de $64,20 \mathrm{kPa}$. As condições do EM foram detector de captura iônica operando por impacto eletrônico e energia de impacto de $70 \mathrm{eV}$; velocidade de varredura 1.000; intervalo de varredura de 0,50 fragmentos s$^{-1}$ e fragmentos detectados na faixa de 40 a $500 \mathrm{Da}$.

A análise quantitativa dos constituintes do óleo essencial foi realizada em um cromatógrafo a gás equipado com detector de ionização de chamas (FID), usando um equipamento Shimadzu GC-17A, sob as seguintes condições operacionais: coluna capilar de sílica fundida ZB5MS com $30 \mathrm{~m}$ x 0,25 mm d.i. x 0,25 $\mu \mathrm{m}$ de filme, usando as mesmas condições do CG-EM. A quantificação dos constituintes foi realizada pela normatização da área (\%). As concentrações dos compostos foram calculadas pela área e colocadas em ordem de eluição do CG.

Os componentes do óleo foram identificados através da comparação de seu espectro de massas com espectros existentes na literatura, ${ }^{20}$ com espectros do banco de dados (NIST21 e NIST107) do equipamento e, também, pela comparação dos índices de retenção com aqueles da literatura. Os índices de retenção de Kovats (IK) foram determinados utilizando uma série homóloga de $n$-alcanos $\left(\mathrm{C}_{8}{ }^{-}\right.$ $\mathrm{C}_{18}$ ) injetados nas mesmas condições cromatográficas das amostras, utilizando a Equação de van den Dool e Kratz. ${ }^{21}$

As médias das variáveis foram submetidas à análise de variância e comparadas pelo teste de Skott-Knott a 5\% de probabilidade.

\section{RESULTADOS E DISCUSSÃO}

Para o teor de óleo essencial observou-se que o armazenamento das folhas por 28 dias proporcionou um aumento de 1,60 para 2,70\% no genótipo POG-002; já no POG-021, não houve diferença significativa para teor de óleo essencial (Tabela 1). Resultados distintos foram obtidos em estudo do efeito do armazenamento de ramos de Ocimum basilicum, nos quais houve decréscimo dos teores de óleo essencial ao longo do armazenamento que, segundo o autor, pode ter sido causado pela volatilização e, assim, ter comprometido a qualidade deste óleo essencial. ${ }^{22}$

Somente aos três dias após armazenamento das folhas secas observou-se diferença entre os dois genótipos de patchouli estudados para o teor de óleo essencial durante o processo de armazenamento (Tabela 1).

Dos compostos encontrados no óleo essencial de patchouli, destaca-se o patchoulol, por sua concentração majoritária nos dois genótipos, variando de 50,67 a 64,02\% no POG-002 e de 51,89 a $58,09 \%$ no POG-021. Estes valores foram superiores aos registrados para o óleo de patchouli da China, 15,00-24,00\% de patchoulol e da Indonésia, 30,00-33,00\% de patchoulol. ${ }^{8}$

Existe uma grande variação quanto à composição química do óleo essencial de patchouli em relação a trabalhos anteriores, e para patchoulol encontraram-se as seguintes concentrações: 28,$8 ;{ }^{4} 32,20 ;{ }^{10}$ 40,$00 ;{ }^{6} 50,66-54,31,{ }^{1}$ e $60,30 \% .{ }^{23}$ Entretanto, também foi encontrado como composto majoritário o $\alpha$-guaieno, com $20,62 \%$. $^{13}$

Observou-se também que, em ambos os genótipos, os componentes principais são os mesmos com diferentes percentagens, o que pode ser consequência da diferente origem dos genótipos, já que variações químicas ocorrem entre plantas de patchouli provenientes de diferentes regiões. ${ }^{12}$

Para o genótipo POG-002, em função do período de armazenamento das folhas, houve diferença significativa na concentração de 9 compostos. A ausência de armazenamento das folhas ( 0 dias) resultou em teor estatisticamente superior aos das folhas armazenadas $(3,7$, 14 e 28 dias) para 5 compostos (cicloseicheleno $0,47 \%, \beta$-cariofileno $2,78 \%, \alpha$-guaieno $8,03 \%$, acifileno $1,47 \%$ e $\alpha$-bulneseno $9,96 \%$ ) no óleo essencial. O contrário foi observado para os compostos pogostol e patchoulol, cujas concentrações no óleo essencial das folhas armazenadas foram significativamente superiores às concentrações das folhas não armazenadas (Tabela 1).

Já para o genótipo POG-021, houve influência significativa do armazenamento das folhas secas na concentração de apenas 2 compostos no óleo essencial, $\alpha$-bulneseno com concentração superior em 14 dias (11,03\%), e germacreno A, superior em 3 e 7 dias de armazenamento $(0,40$ e $0,41 \%)$ (Tabela 1$)$.

Assim como para alguns compostos do óleo essencial de patchouli, foi observado efeito do armazenamento dos ramos de Ocimum basilicum sobre a composição do seu óleo essencial, ocorrendo aumento dos teores de eugenol e linalol durante o armazenamento. ${ }^{21}$ Para a mesma espécie, em estudo sobre o efeito do período de secagem de folhas e inflorescências no óleo essencial, constatou-se que o teor de linalol aumentou de 45,18 nas folhas frescas para 86,80\% após 5 dias de secagem, e de 80,68 nas inflorescências frescas para $92,62 \%$ após 11 dias de secagem. ${ }^{24}$

Em folhas secas não armazenadas do genótipo POG-002, observaram-se concentrações estatisticamente superiores à do genótipo POG021 para os compostos: cicloseicheleno $(0,47 \%), \beta$-cariofileno $(2,78 \%)$, $\alpha$-guaieno $(8,03 \%)$, acifileno $(1,47 \%)$ e longicanfenilona $(1,13 \%)$ (Tabela 1). Apenas o patchoulol apresentou percentagem estatisticamente superior no genótipo POG-021 para folhas não armazenadas.

No genótipo POG-021 o armazenamento das folhas proporcionou concentração estatisticamente superior em relação ao POG-002 para os compostos $\alpha$-guaieno, acifileno, $\alpha$-bulneseno e germacreno A (3, 7, 14 e 28 dias), $\beta$-cariofileno ( 3,14 , e 28), cicloseicheleno, seicheleno, $\alpha$-humuleno, $\alpha$-patchouleno e allo-aromadrendeno (14 e 28 dias), e $\beta$-patchouleno (28 dias para) (Tabela 1).

\section{CONCLUSÕES}

O armazenamento das folhas secas de patchouli por 28 dias proporcionou aumento do teor de óleo essencial apenas do genótipo 
Tabela 1. Valores médios dos teores dos compostos do óleo essencial de patchouli (P. cablin), genótipos POG-002 e POG-021, em função do tempo de armazenamento

\begin{tabular}{|c|c|c|c|c|c|c|}
\hline \multirow{2}{*}{ Composto } & \multirow[b]{2}{*}{ IK } & \multicolumn{5}{|c|}{ Tempo de armazenamento } \\
\hline & & as & 3 dias & 7 dias & 14 dias & 28 dias \\
\hline & & \multicolumn{5}{|c|}{ POG-002 } \\
\hline houleno & 1380 & 2,23 a A & 2,28 a A & 2,05 a A & & $1,53 \mathrm{~b} \mathrm{~A}$ \\
\hline eno & 1390 & 0,59 a $\mathrm{A}$ & 0,45 a A & 0,55 a $\mathrm{A}$ & a A & 0,39 a $\mathrm{A}$ \\
\hline icloseichel & 1407 & 0,47 a $\mathrm{A}$ & 0,30 a B & 0,29 a B & 0,27 b B & $0,15 \mathrm{~b} \mathrm{C}$ \\
\hline -cariofileno & 1419 & 2,78 a $A$ & $1,74 \mathrm{~b} \mathrm{~B}$ & 1,74 a B & $1,70 \mathrm{~b} B$ & $1,33 \mathrm{~b} \mathrm{~B}$ \\
\hline & 1439 & 8,03 a $\mathrm{A}$ & B & b B & $\mathrm{B}$ & b B \\
\hline eicheleno & 1446 & 4,09 a A & 4,07 a $\mathrm{A}$ & & & b A \\
\hline & 1454 & 0,39 a A & & a A & b A & b A \\
\hline$\alpha-\mathrm{pa}$ & 1456 & 2,50 a $\mathrm{A}$ & 2,56 a $\mathrm{A}$ & 2,39 a $\mathrm{A}$ & $2,46 \mathrm{~b} \mathrm{~A}$ & b A \\
\hline $\begin{array}{l}\text { allo- } \\
\text { aromadrendeno }\end{array}$ & 1460 & A & A & & & \\
\hline acifi & 1501 & 1,47 a $\mathrm{A}$ & $\mathrm{B}$ & B & $\mathrm{B}$ & b B \\
\hline$\alpha$-bu & 1509 & 9,96 a $A$ & $\mathrm{~B}$ & & & $b \mathrm{~B}$ \\
\hline & 1509 & A & & & & b B \\
\hline ililona & 1563 & B & 1,03 & $\mathrm{~B}$ & & A \\
\hline 3-atlantol & 1608 & 0,74 a $\mathrm{A}$ & 0,87 a $\mathrm{A}$ & 0,92 a A & 1,05 a A & 1,34 a A \\
\hline & 1653 & 3,39 a $\mathrm{B}$ & 4,28 a $\mathrm{A}$ & a A & a A & a A \\
\hline & 1658 & & & & 61 & a \\
\hline \multirow[t]{2}{*}{$\begin{array}{l}\text { Teor de óleo } \\
\text { essencial (\%) }\end{array}$} & & 3 & & & & 2 \\
\hline & & \multicolumn{5}{|c|}{ POG-021 } \\
\hline & 1380 & 2 & 2, & 2,11 a A & & a A \\
\hline & 1390 & & & & & a A \\
\hline cicloseicheleno & 1407 & b A & 0,36 a $\mathrm{A}$ & a A & a A & a A \\
\hline$\beta$-ca & 1419 & 2 , & 2,4 & a A & a A & a A \\
\hline & 1439 & 6 , & & a A & & $1 \mathrm{a} A$ \\
\hline Seicheleno & 1446 & 4,43 a $\mathrm{A}$ & 4,69 a $\mathrm{A}$ & a A & $\mathrm{a} A$ & a A \\
\hline$\alpha$-hu & 1454 & a A & a A & a A & a A & a A \\
\hline$\alpha$-patchouleno & 1456 & 2,77 a A & 2,92 a A & 2 a A & 8 a A & 3,02 a A \\
\hline $\begin{array}{l}\text { allo- } \\
\text { aromadrenc }\end{array}$ & 1460 & 0,87 a $\mathrm{A}$ & 0,93 a $\mathrm{A}$ & a A & a A & 0 \\
\hline acifileno & 1501 & 1,2 & 1,46 a A & a A & $\mathrm{a} A$ & a A \\
\hline$\alpha$-bulneseno & 1509 & 9,02 a B & 9,61 a B & 8,14 a B & 11,03 a A & 9,01 a B \\
\hline germacreno A & 1509 & 0,30 a B & 0,40 a $\mathrm{A}$ & 0,41 a $\mathrm{A}$ & 0,34 a B & 0,33 a $\mathrm{B}$ \\
\hline longicanfenilona & 1563 & $0,72 \mathrm{~b} \mathrm{~A}$ & $0,72 \mathrm{~b} \mathrm{~A}$ & $0,77 \mathrm{~b} \mathrm{~A}$ & $0,81 \mathrm{~b} \mathrm{~A}$ & $0,83 \mathrm{~b} \mathrm{~A}$ \\
\hline$\beta$-atlantol & 1608 & 0,91 a A & 1,13 a $\mathrm{A}$ & 0,89 a $\mathrm{A}$ & & $0,90 \mathrm{~b} \mathrm{~A}$ \\
\hline & 1653 & 3,87 a A & 3,95 a A & 3,98 a A & 3,45 b A & $383 \mathrm{~h}$ \\
\hline patchoulol & & & & & & \\
\hline
\end{tabular}

Teor de óleo

essencial $(\%)$

2,13 a A 2,27 a A 1,80 a A 2,33 a 2,53 a A

Médias seguidas das mesmas letras minúsculas, entre genótipos, e maiúsculas, entre os tempos de armazenamento, não diferem entre si, pelo teste de ScottKnott $(\mathrm{p} \leq 0,05)$. IK= Índice de Kovats

POG-002. O armazenamento também proporcionou concentração significativamente superior no genótipo POG-002, em relação ao POG-021, para os compostos pogostol e patchoulol, e inferior para os seguintes compostos: $\beta$-patchouleno, cicloseicheleno, $\beta$-cariofileno, $\alpha$-guaieno, seicheleno, $\alpha$-humuleno, $\alpha$-patchouleno, allo-aromadrendeno, acifileno, $\alpha$-bulneseno e germacreno A. Há variabilidade para teor de patchoulol no óleo essencial dos genótipos, que neste trabalho foram superiores aos óleos produzidos em outros países.

\section{MATERIAL SUPLEMENTAR}

Está disponível em http://quimicanova.sbq.org.br, na forma de arquivo PDF, com acesso livre. Compreende os cromatogramas, estrutura dos principais componentes do óleo essencial e foto da espécie estudada.

\section{AGRADECIMENTOS}

À FAPITEC/SE, à RARO'S Agroindústria de Produtos Aromáticos S.A. e ao CNPq pelo financiamento da pesquisa, e à CAPES pela bolsa de mestrado do primeiro autor.

\section{REFERÊNCIAS}

1. Singh, M.; Sharma, S.; Ramesh, S.; Ind. Crop. Prod. 2002, 16, 101.

2. Salerno, A. R.; Rebelo, A. M.; Silva Junior, A. A.; Agropecuária Catarinense 2004, 17, 46.

3. Khare, C. P.; Indian Medicinal Plants: an illustrated dictionary, Springer: New Delhi, 2007.

4. Wei, A.; Shibamoto, T.; J. Agric. Food Chem. 2007, 55, 1737.

5. Pavela, R.; Fitoterapia 2005, 76, 691.

6. Zhu, B. C. R.; Henderson, G.; Yu, Y.; Laine, R. A.; J. Agric. Food Chem. 2003, 51, 4585 .

7. Guenther, E.; The Essential Oils: individual essential oils of the plant families Rutaceae and Labiatae, Krieger Publishing Company: Malabar, 1972.

8. Milchard, M. J.; Clery, R.; Dacosta N.; Esdale, R.; Flowerdew, M.; Gates, L.; Moss, N.; Moyler, D. A.; Sherlock, A.; Starr, B.; Webb, J.; Wootten, J.; Wilson, J. J.; Perf. Flavor. 2004, 29, 28.

9. Bizzo, H. R.; Hovell, A. M. C.; Rezende, C. M.; Quim. Nova 2009, $32,588$.

10. Bure, C. M.; Sellier N. M.; J. Essent. Oil. Res. 2004, 16, 17.

11. Anonis, D. P.; Perf. Flavor. 2006, 31, 36.

12. Hu, L. F.; Li, S. P.; Cao, H.; Liu, J. J.; Gao, J. L.; Yang, F. Q.; Wang, Y. T.; J. Pharm. Biomed. Anal. 2006, 42, 200.

13. Tsai, Y.; Hsu, H.; Yang, W.; Tsai, W.; Chen, C.; Watanabe, T.; Fitoterapia 2007, 78, 7 .

14. Vieira, R. F.; Costa, T. S. A. Em Recursos Genéticos Vegetais; Nass, L., org.; Embrapa Recursos Genéticos e Biotecnologia: Brasília, 2006, cap. 10.

15. Deguerry, F.; Pastore, L.; Wu, S.; Clark, A.; Chappell, J.; Schalk, M.; Arch. Biochem. Biophys. 2006, 454, 123.

16. Silva, M. A. S.; Ehlert, P. A. D.; Ming, L. C.; Marques, M. O. M.; Acta Hortic. 2004, 629, 145.

17. Ramachandra, K. M.; Vasundhara, M.; Farooqi, A. A.; Srinivasappa, K. N.; J. Spices Arom. Crops 2003, 12, 43.

18. Ram, M.; Ram, D.; Singh, S.; Naqvi, A. A.; Kumar, S.; J. Med. Arom. Plant Sci. 1999, 21, 358.

19. Luo, J. P.; Liu, Y. P.; Feng, Y. F.; Guo, X. L.; Cao, H.; Yao Xue Xue Bao 2003, 38, 307 .

20. Adams, R. P.; Identification of Essential Oil Components by Gas Chromatography/Mass Spectroscopy, Allured Publishing Corporation: Illinois, 2007.

21. van den Dool, H.; Kratz, P. D. J.; J. Chromatogr. 1963, 11, 463.

22. Silva, F.; Santos, R. H. S.; Andrade, N. J.; Barbosa, L. C. A.; Casali, V. W. D.; Lima, R. R.; Passarinho, R. V. M.; Pesq. Agrop. Bras. 2005, 40, 323.

23. Bunrathep, S.; Lockwood, G. B.; Songsak, T.; Ruangrungsi, N.; Sci. Asia 2006, 32, 293.

24. Carvalho Filho, J. L. S.; Blank, A. F.; Alves, P. B.; Ehlert, P. A. D.; Melo, A. S.; Cavalcanti,, S. C. H.; Arrigoni-Blank, M. F.; Silva-Mann, R.; Rev. Bras. Farmacogn. 2006, 16, 24. 


\section{INFLUÊNCIA DO ARMAZENAMENTO DE FOLHAS SECAS NO ÓLEO ESSENCIAL DE PATCHOULI}

(Pogostemon cablin BENTH.)

Trícia Cavalcanti Pergentino de Sant'ana, Arie Fitzgerald Blank*, Sylvia Dantas Vieira e Maria de Fátima Arrigoni-Blank Departamento de Engenharia Agronômica, Universidade Federal de Sergipe, Av. Mal. Rondon, s/n, 49100-000-São Cristóvão - SE, Brasil Hugo César R. de Jesus e Péricles Barreto Alves

Departamento de Química, Universidade Federal de Sergipe, Av. Mal. Rondon, s/n, 49100-000 São Cristóvão - SE, Brasil
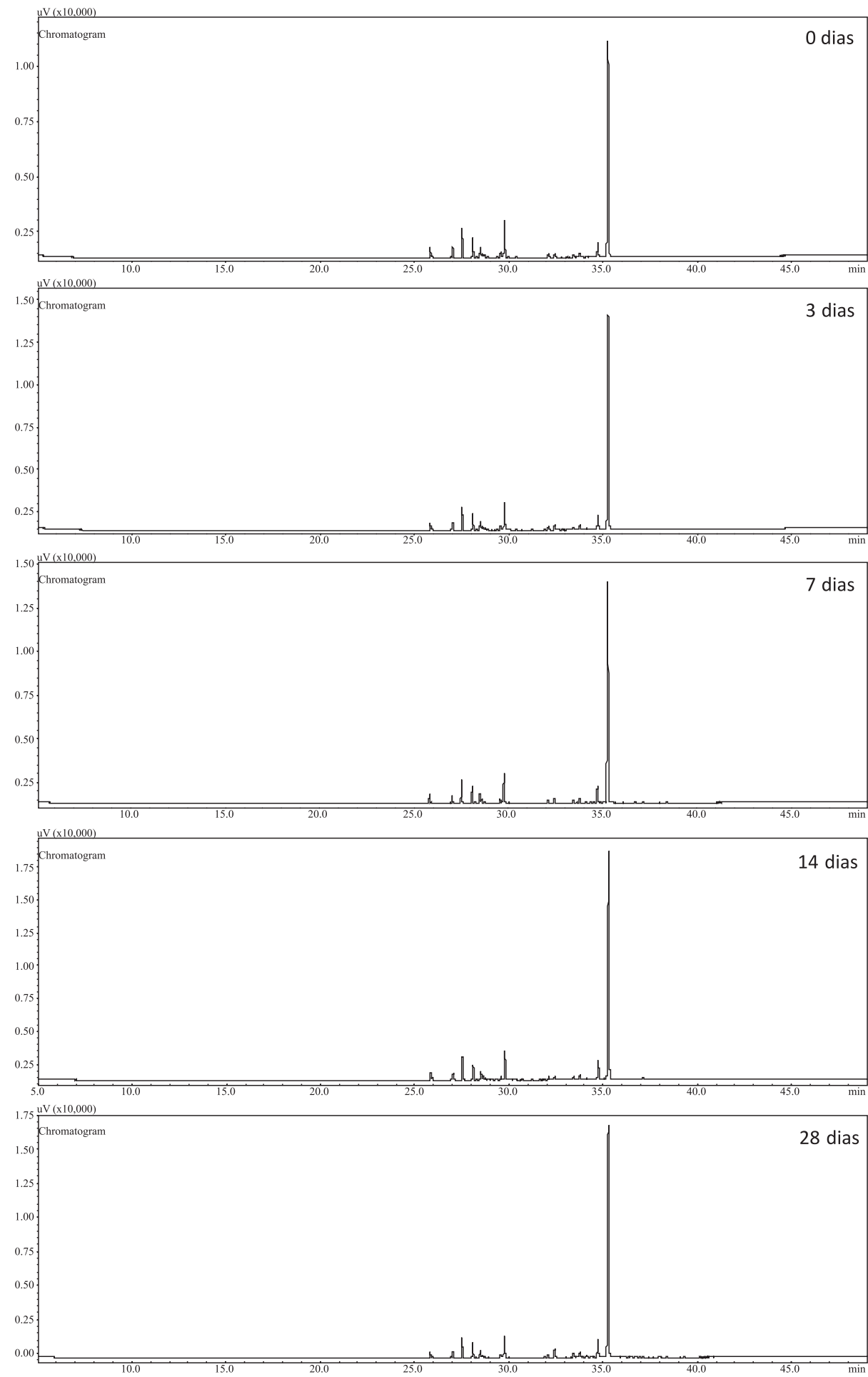

Figura 1S. Cromatogramas obtidos por CG/DIC de amostras de óleo essencial extraído de folhas secas de patchouli POG-002 armazenadas por 0, 3, 7, 14 e 28 dias 

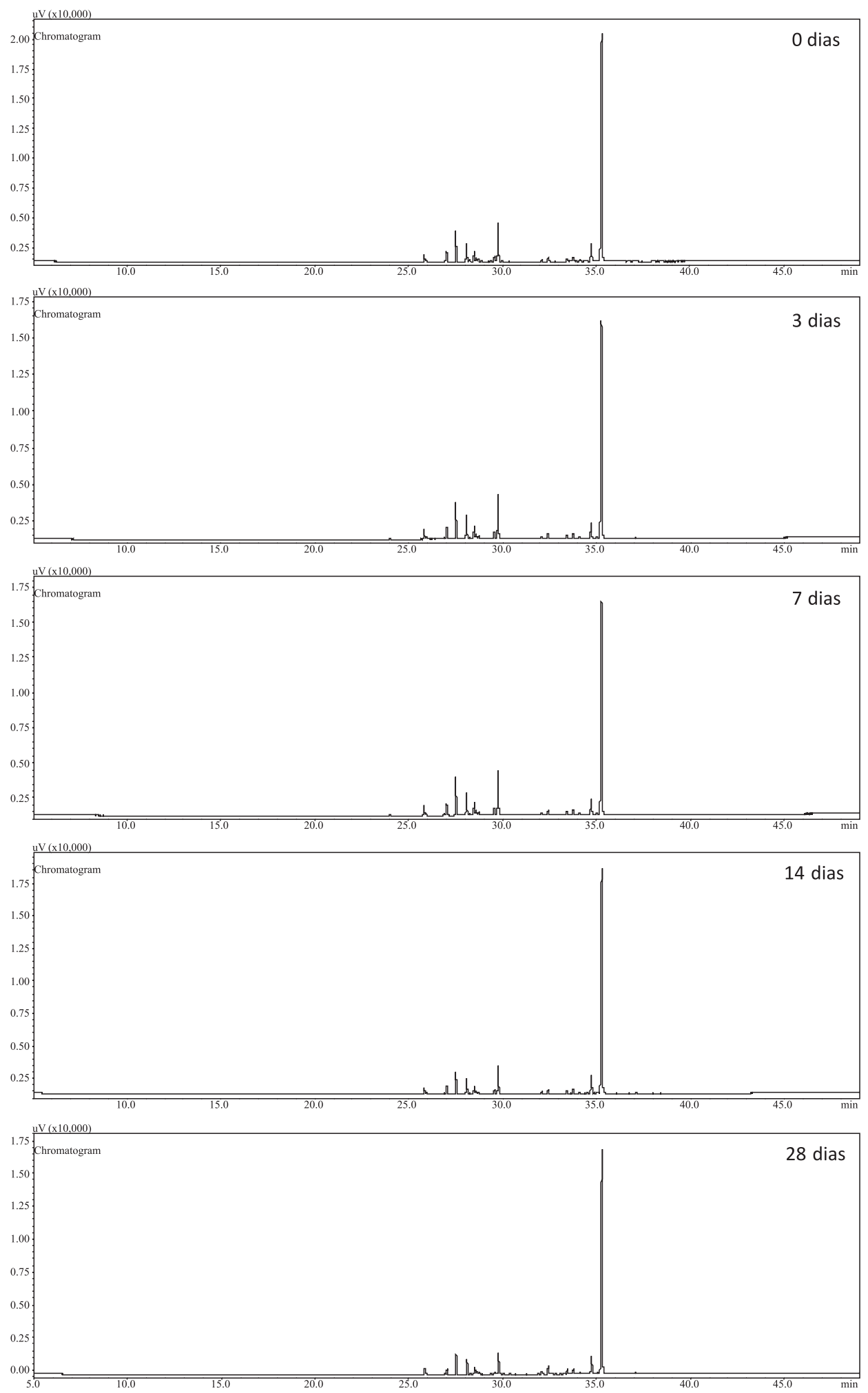

Figura 2S. Cromatogramas obtidos por CG/DIC de amostras de óleo essencial extraído de folhas secas de patchouli POG-021 armazenadas por 0, 3, 7, 14 e 28 dias 

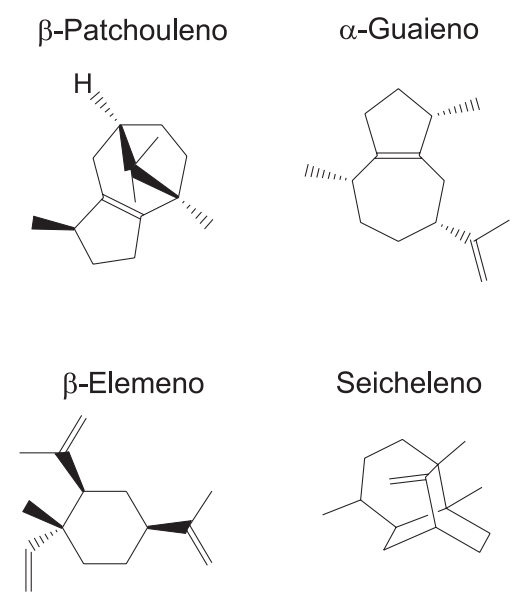

Cicloseicheleno

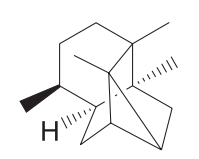

$\beta$-Cariofileno



$\alpha$-Humuleno

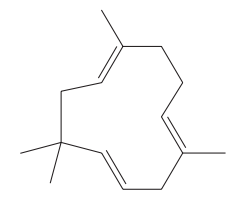

$\alpha$-Patchouleno

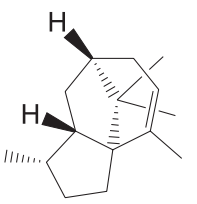

Allo-Aromadendreno

$\beta$-Atlantol<smiles>C=C1CC[C@H](C)[C@H](C)[C@H]1[C@H]1CCC(C)[C@H]1C</smiles><smiles>C=C(C)[C@@H]1CC[C@@H](C)[C@H]2CCC(C)=C2C1</smiles><smiles>C=C(CC(O)C=C(C)C)C1CC=C(C)CC1</smiles>

\section{Germacreno A}<smiles>C=C(C)C1CC=C(C)CCC=C(C)CC1</smiles>

Longicanfenilona<smiles>C=C(C)[C@@H]1CCC(C)=C2CC[C@@H](C)[C@H]2C1</smiles>

Óxido de cariofileno<smiles>C=C1CC[C@H]2O[C@H]2C[C@@H]2[C@@H]1CC2(C)C</smiles><smiles>CC1(C)CCCC2(C)C(=O)C3CCC1C32</smiles>

Pogostol<smiles>C=C(C)C1CCC(C)(O)C2CCC(C)C2C1</smiles>

Patchoulol

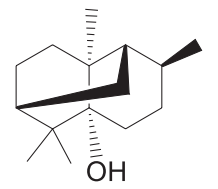

Figura 3S. Estruturas dos componentes do óleo essencial extraído de folhas secas de patchouli

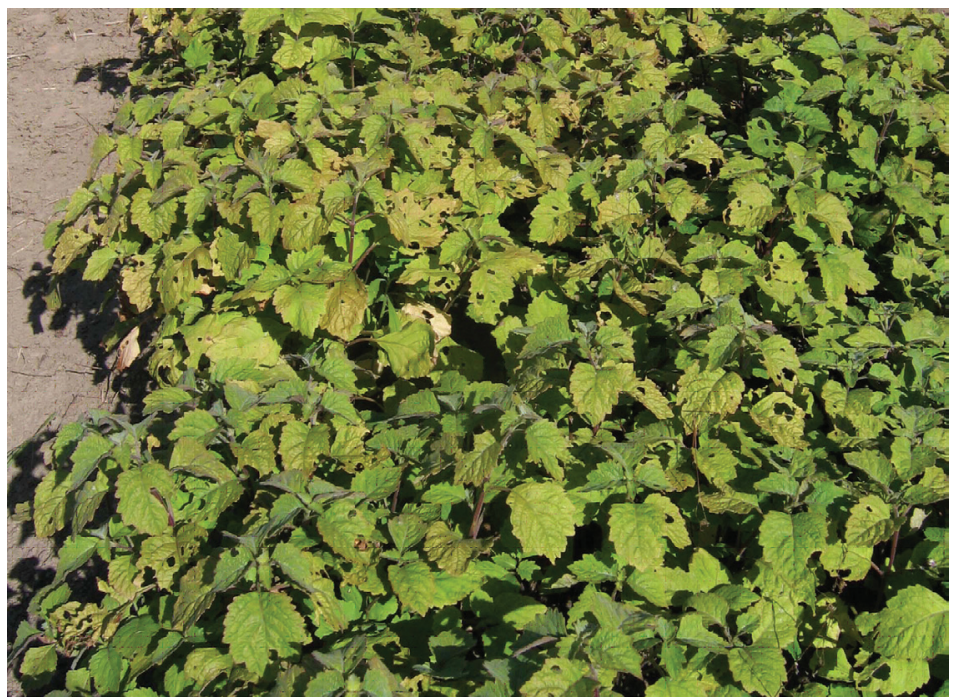

Figura 4S. Foto de plantas de patchouli (Pogostemon cablin L.) cultivados em campo 